\title{
Risque, crise et sécurité sanitaires des aliments
}

Oléagineux, Corps Gras, Lipides. Volume 7, Numéro 5, 385, Septembre - Octobre 2000, Editorial

Auteur(s) : Jean-Claude ICART, .

Résumé : Amené par la crise, un ré-examen des stratégies d'acteur est aujourd'hui à l'œuvre sur les questions de sécurité sanitaire. Des outils, des dispositifs, des programmes de recherche, des approches nouvelles sont développés ou en cours de développement et sur lesquels, pour quelquesuns d'entre eux, nous avons souhaité faire le point.

\section{ARTICLE}

Amené par la crise, un ré-examen des stratégies d'acteur est aujourd'hui à l'œuvre sur les questions de sécurité sanitaire. Des outils, des dispositifs, des programmes de recherche, des approches nouvelles sont développés ou en cours de développement et sur lesquels, pour quelques-uns d'entre eux, nous avons souhaité faire le point.

En haut de l'agenda des pouvoirs publics, depuis presque une décennie, ce thème a débouché, avec la création de l'AFSSA, sur la définition d'une politique générale en matière d'évaluation du risque ${ }^{1}$. Centrée autour de la mise en œuvre du principe de précaution et une stricte séparation entre contrôleur et contrôlé (voir l'article de P. Hubert), ce cadre demanderait encore que soient mieux définies les relations d'échange d'informations entre opérateurs et contrôleurs. De même si, face à la complexité du problème (7 000 substances concernées), une méthodologie de traitement des aspects toxicologiques a été définie (voir l'article de J.-F. Narbonne), elle nécessiterait toutefois encore une clarification des responsabilités entre scientifiques et décideurs administratifs et politiques ${ }^{2}$.

L'engagement des opérateurs industriels, l'auto-contrôle de leurs process fondé sur les principes de la méthode HACCP (obligatoire depuis 1998), passe également aujourd'hui par la mise en place de cellule de gestions de crises, le lancement de différents programmes (en microbiologie, sur la question des emballages) et parallèlement par le développement de la traçabilité. Face à la multiplication et à l'allongement des réseaux de circulation des matières premières et des produits, cette question de leur sécurité est devenue primordiale. Ses incidences prévisibles (du fonctionnement du commerce international aux transformations des process par le choix de nouvelles matières premières) sont nombreuses et encore loin de s'être toutes concrétisées.

En particulier, avec le développement de la traçabilité, c'est la participation de l'agriculture à la qualité sanitaire des aliments qui est en passe de s'approfondir. La nécessité d'une approche « filière " (la fameuse gestion de la fourche à la fourchette) est ici soulignée (Denis Delplancke) : soit l'ouverture d'un champ de recherche dont on peut imaginer ou souhaiter, comme le fait Jean- 
François Narbonne ${ }^{3}$, que le terme soit, dans le domaine des contaminants, la formalisation et la mise en place d'une démarche HACCP de « filière ».

Des problèmes liés aux risques sanitaires, la crise s'est aujourd'hui élargie à la mise en cause des principes de production qui les ont suscités. Les différentes plate-formes de débat ${ }^{4}$ ou enquêtes ${ }^{5}$ constituées ces derniers mois, le confirment.

Lié à des dimensions symboliques et culturelles incontournables, "l'aliment " relève de besoins complexes. Sans être le seul, le développement de réseaux d'informations et d'outils autour de la sécurité des produits est aujourd'hui un de ces axes d'action prioritaire.

${ }^{1}$ Voir Chevassus-au-Louis B (2000). L'analyse du risque alimentaire : quels principes, quels modèles, quelles organisations pour demain, http://www.oecd.org/subject/biotech/ChevassusauLouisfr.pdf

${ }^{2}$ Voir sur ce sujet Gérard Pascal (1999). Faut-il intégrer des seuils de préoccupation toxicologique dans les réglementations ? La Recherche, Octobre.

${ }^{3}$ Communication orale.

${ }^{4}$ Voir les différentes tables rondes des états généraux de l'alimentation : http://www.agriculture.gouv.fr/ega/index.htm

${ }^{5}$ Voir l'enquête Inca (2000). Enquête individuelle et nationale sur les consommations alimentaires. Paris : Éditions TEC\&DOC. 
